\title{
New Data from the ISOIS Instrument Suite on Parker Solar Probe
}

\author{
E. R. Christian ${ }^{a, *}$ C. M. S. Cohen ${ }^{b}$, A. C. Cummings ${ }^{b}$, A. J. Davis ${ }^{b}$, M. I. Desai ${ }^{c}$, G. A. \\ de Nolfo $^{a}$, J. Giacalone ${ }^{d}$, M. E. Hill ${ }^{e}$, C. J. Joyce ${ }^{f}$, A. W. Labrador ${ }^{b}$, R. A. Leske ${ }^{b}$, W. \\ H. Matthaeus ${ }^{g}$, D. J. McComas ${ }^{f}$, R. L. McNutt Jr. ${ }^{e}$, R. A. Mewaldt ${ }^{b}$, D. G. Mitchell ${ }^{e}$, J. \\ G. Mitchell ${ }^{a, h}$, J. S. Rankinf, E. C. Roelof ${ }^{e}$, N. A. Schwadron ${ }^{i}$, E. C. Stone ${ }^{b}$, J. R. \\ Szalay ${ }^{f}$, and M.E. Wiedenbeck, ${ }^{j, b}$
}

a Energetic Particle Laboratory, NASA Goddard Space Flight Center, Code 672 8800 Greenbelt Rd., Greenbelt, MD 20771, USA

${ }^{b}$ Space Radiation Laboratory, California Institute of Technology 1200 E. California Blvd., Pasadena, CA 91125, USA

c Southwest Research Institute 6220 Culebra Rd., San Antonio, TX 78238, USA

${ }^{d}$ Lunar and Planetary Laboratory, University of Arizona PO Box 210092, Tuscon, AZ 85721, USA

e Johns Hopkins University Applied Physics Laboratory 11100 Johns Hopkins Rd., Laurel, MD 20723, USA

${ }^{f}$ Dept. of Astrophysical Sciences, Princeton University 4 Ivy Lane, Princeton, NJ 08544, USA

$g$ Department of Physics and Astronomy, University of Delaware, Newark DE 19716

${ }^{h}$ Department of Physics, George Washington University 725 21st St. NW, Washington DC 20052, USA

i Space Science Center, University of New Hampshire 8 College Rd., Durham, NH 03824, USA

j NASA Jet Propulsion Laboratory 4800 Oak Grove Dr., La Cañada, CA 91109

*Presenter

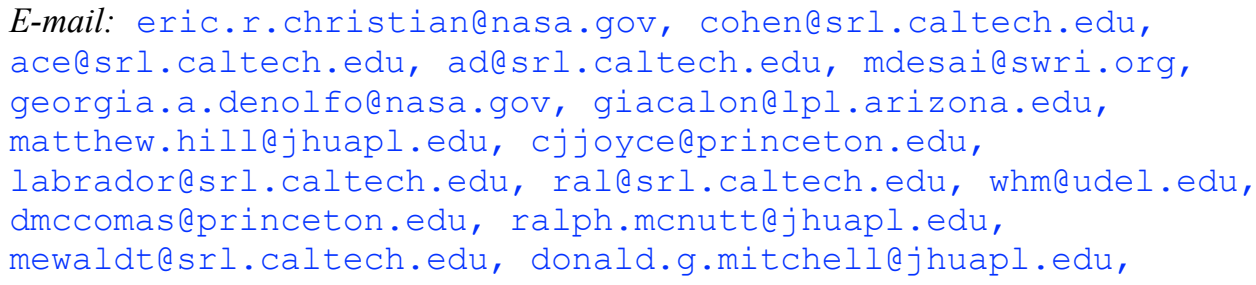


john.g.mitchell@nasa.gov, jsrankin@princeton.edu,
edmond.roelof@jhuapl.edu, nschwadron@guero.sr.unh.edu,
jszalay@princeton.edu, mark.e.wiedenbeck@jpl.nasa.gov

NASA's Parker Solar Probe (PSP) mission's first seven orbits include perihelia as close as $\sim 11$ million $\mathrm{km}(\sim 16$ solar radii), much closer to the Sun than any prior human-made object. Onboard PSP, the Integrated Science Investigation of the Sun (IS@IS) instrument suite makes groundbreaking measurements of solar energetic particles (SEPs). Here we discuss the near-Sun energetic particle radiation environment over PSP's first two and a half years, which reveal where and how energetic particles are energized and transported. We find a great variety of energetic particle events accelerated both locally and remotely. These include stream and co-rotating interaction regions (SIRs and CIRs), "impulsive" SEP events driven by magnetic reconnection near the Sun, and events related to Coronal Mass Ejections (CMEs). These IS@IS observations made close to the Sun provide critical information for investigating the near-Sun transport and energization of solar energetic particles, which has been difficult to resolve from prior observations. The Parker Solar Probe IS@IS data are made public soon after receipt at Earth (which can be many months after the observations). We will also discuss how to get access to the data.

37th International Cosmic Ray Conference (ICRC 2021)

July 12th-23rd, 2021

Online-Berlin, Germany 


\section{Introduction}

The Parker Solar Probe mission launched on 2018 August 12, and has had eight successful orbits around the Sun. The perihelion distance gets smaller with every Venus encounter, and Parker has already completed four of the seven planned encounters, which have moved the perihelion to nearly $16 \mathrm{R}_{\odot}(10.4$ million $\mathrm{km})$. One of the Parker instrument suites for this extraordinary exploration of the inner heliosphere is the Integrated Science Investigation of the Sun (IS@IS), which measures suprathermal and energetic ions and electrons. This paper will give selected highlights of the IS@IS data from the first seven orbits.

\section{Observations}

The IS@IS suite has two instruments, the Energetic Particle Instrument - Low Energy (EPILo, and the Energetic Particle Instrument - High Energy (EPI-Hi). EPI-Lo consists of eighty apertures that sample a complete hemisphere $(2 \pi)$ nominally in the ram direction. EPI-Lo measures protons from a few tens of $\mathrm{keV}$ to a few $\mathrm{MeV}$, ions from tens of $\mathrm{keV} /$ nucleon to more than $500 \mathrm{keV} /$ nucleon, and electrons from less than $20 \mathrm{keV}$ to nearly $1 \mathrm{MeV}$. EPI-Hi has a doubleended Low-Energy Telescope (LET) nominally pointing approximately sunward-antisunward but skewed in the direction of the Parker Spiral, a single-ended LET perpendicular to that, and a double-ended High-Energy Telescope (HET) also pointed nominally sunward-antisunward. EPIHi measures protons from about $1 \mathrm{MeV}$ to more than $100 \mathrm{MeV}$, ions between about 1 and 200 $\mathrm{MeV}$ /nucleon, and electrons between 0.5 and $6 \mathrm{MeV}$. IS@IS is described in detail in McComas et al. 2016 [1].

\subsection{Orbit Summary}

Summaries of the first seven orbits of Parker Solar Probe are shown in Figure 1. EPI-Hi proton data (1-2 MeV) and EPI-Lo data (30-200 keV) are plotted on the outside and inside of the orbit trajectories, respectively. Note that the length of the bars extending from the orbital path and the color display the same information, the observed counting rates of the energetic protons. Also, EPI-Hi was turned off during Orbit 4, so only EPI-Lo is displayed for that orbit.

One thing that stands out from these plots is that many of the events seen in EPI-Hi are smaller or absent in EPI-Lo and vice versa. This indicates that few events have power laws or other monotonically decreasing spectra over the entire IS๑IS energy range.

\subsection{Stream Interaction Regions and Co-rotating Interaction Regions}

As is typical of solar minimum, many of the increases in energetic particle intensity seen by Parker Solar Probe during the early part of the mission are due to stream interaction regions (SIRs). These are compression regions caused by fast solar wind streams overtaking slower solar wind. When the fast and slow streams are long-lived, these regions repeat over solar rotations and are known as Co-rotating Interaction Regions (CIRs). Parker's orbit gives a tremendous opportunity for the study of how these regions develop and how they accelerate suprathermal and energetic particles. 

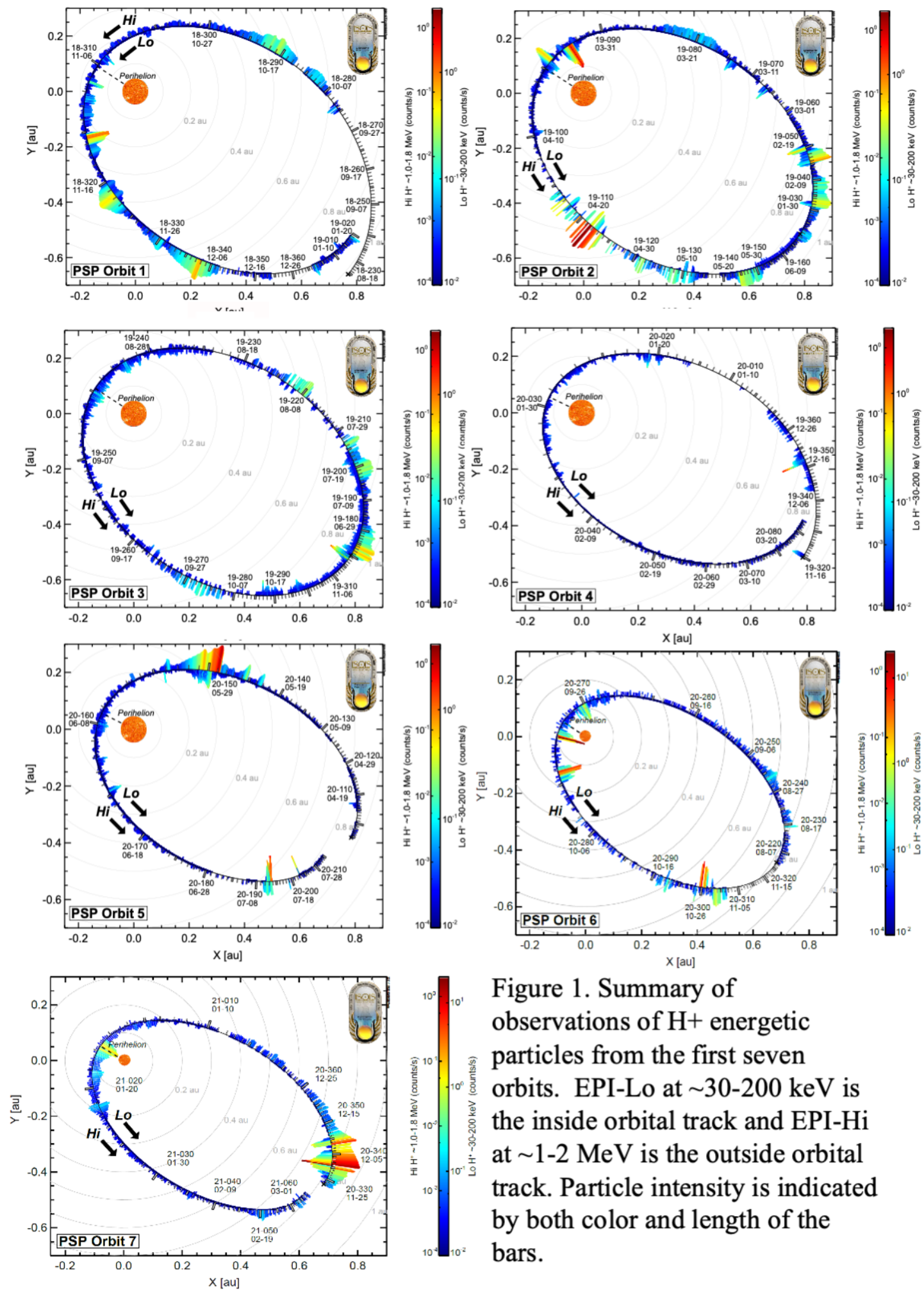

Figure 1. Summary of observations of $\mathrm{H}+$ energetic particles from the first seven orbits. EPI-Lo at $\sim 30-200 \mathrm{keV}$ is the inside orbital track and EPI-Hi at $\sim 1-2 \mathrm{MeV}$ is the outside orbital track. Particle intensity is indicated by both color and length of the bars. 

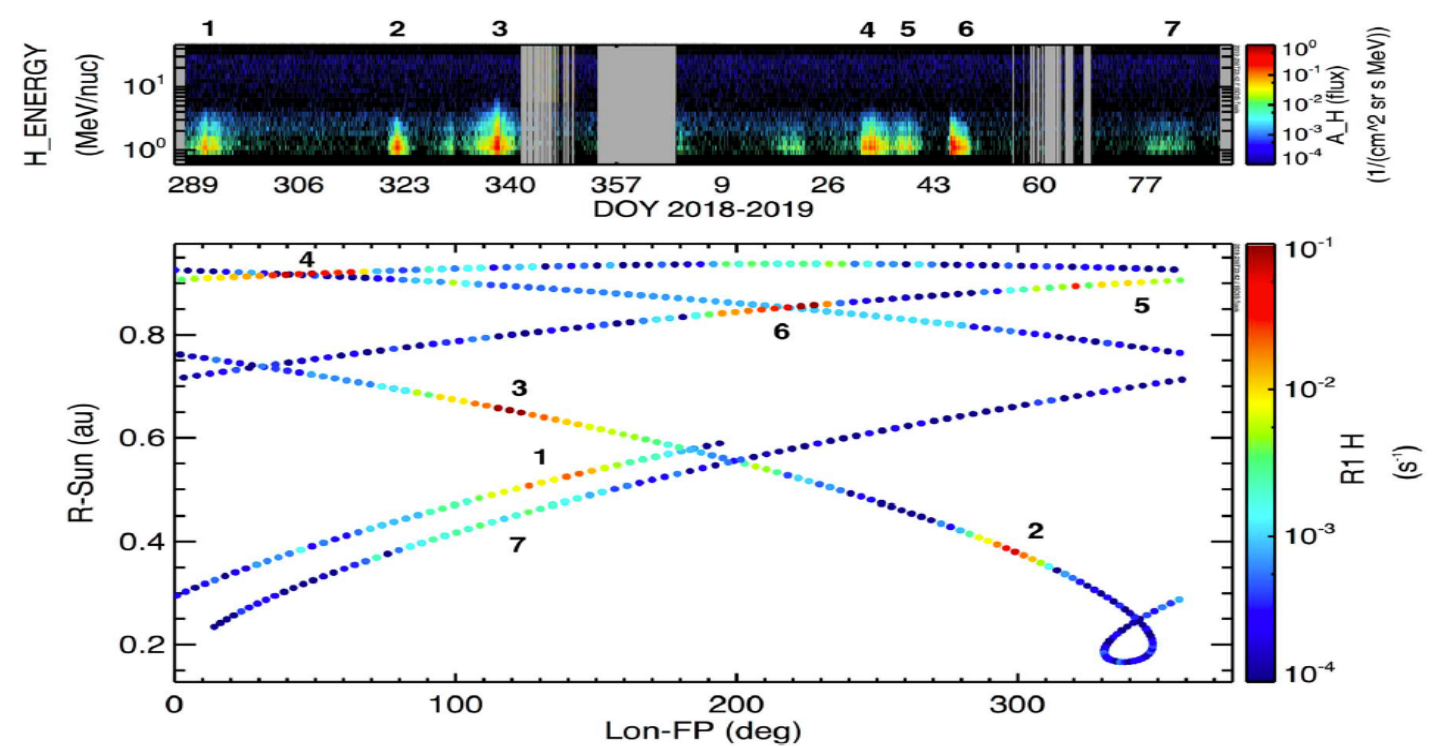

Figure 2. (Top panel) EPI-Hi LETA proton intensity spectrograms. The gray regions are data gaps. The seven increases identified with SIRs are labeled (1-7) at the top. (Bottom Panel) 1-2 $\mathrm{MeV}$ proton counting rates as a function of the longitude of the magnetic footpoint for Parker (calculated assuming a Parker spiral and $\mathrm{Vsw}=400 \mathrm{~km} / \mathrm{s}$ ) and the radial distance from the Sun. The labels correspond to the events from the top panel.

Increases in energetic particles due to SIRs have been studied since the beginning of the space age [2 and references therein]. There were seven proton increases identified as SIRs that were observed by IS@IS during the first two orbits (see Figure 2) [3, 4]. Four of these occur at distances less than about 0.65 au, nearly doubling the sample of these events seen in the inner heliosphere [5]. Increases 1 and 3, on successive passes, appear to be the same interaction region. However, event 7, which has a footpoint at the same longitude, occurs more than 100 days later, after at least two passes that don't show any increase and so is probably unrelated to events 1 and 3. There are no obvious solar wind shocks or compression regions near these events, the particle distributions are isotropic, and there is no clear velocity dispersion, which probably means that these increases are not due to local acceleration or a single distant acceleration event. It seems likely that the spacecraft is passing through regions that have already been filled with energetic particles.

In addition, a detailed study of time evolution of event 2 [6] show that the energy spectra are inconsistent with acceleration at a distant shock and are likely due to non-localized energization at pre-shock compression regions, which is likely to be important for the understanding of the acceleration process.

\subsection{Solar Energetic Particles}

All of the Solar Energetic Particle events observed by Parker except one have been small. There is still a lot that can be learned from the study of these events. The variation of these early SEP events in composition has been remarkable. One very intriguing series of events occurred in the middle of 2020 during orbit 5 [7], which are labeled events 3, 4, and 5 on Figure 3. Even though these events occurred within a few days and apparently from the same active region, their composition varied widely. The observed $\mathrm{He} / \mathrm{H}$ ratio integrated over the three events between about 1 and $10 \mathrm{MeV} /$ nucleon was 0.0033 (event 3), 0.18 (event 4), and 0.016 (event 5), and so 
differed by more than a factor of fifty. There is also evidence that the ${ }^{3} \mathrm{He} /{ }^{4} \mathrm{He}$ showed significant differences, although the statistics were limited. This is an important puzzle that will be one of the focus areas for IS@IS over this mission.

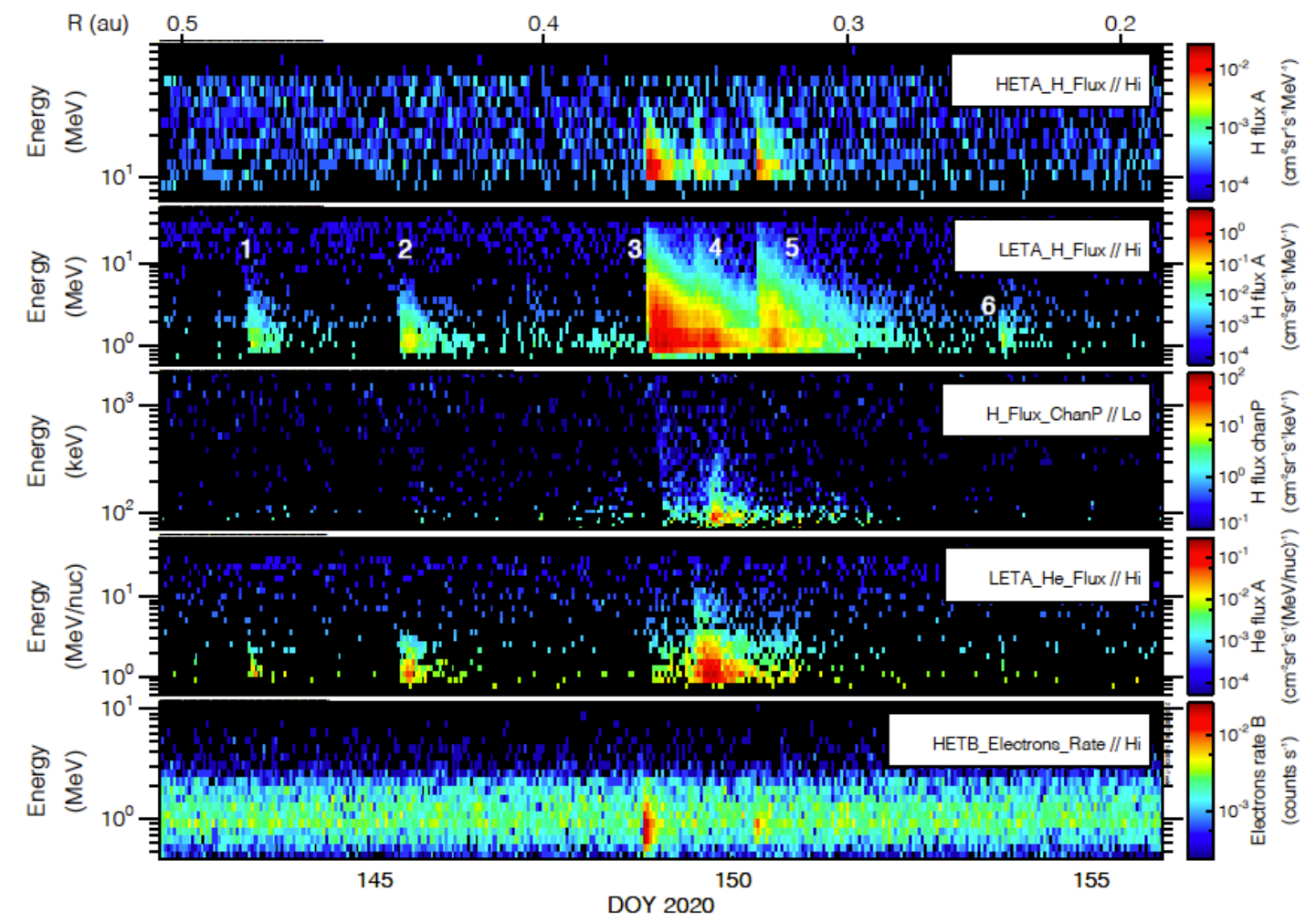

Figure 3. Energy spectrograms for EPI-Hi HETA protons (top panel), EPI-Hi LETA protons (second panel), EPI-Lo (third panel), EPI-Hi LETA helium, and EPI-Hi HETB electrons. Events numbers are given at the top of the second panel.

\subsection{Electrons}

The quiet Sun has allowed ISৎIS to look at electron events that are smaller than any earlier study [8]. During the first Parker perihelion pass, there were no statistically significant electron events. The second perihelion pass was more active, with four discernible events (Figure 4). The first appears to be associated with a magnetic structure that included a current sheet. The last three events during this pass, with Parker at about 0.19 au, are associated with Type III radio emissions. However, the delay between the radio bursts and the observed electron increases is variable even though all three events occur within 12 hours, and Parker hadn't moved very much. The combination of IS@IS energetic electron measurements close to the Sun and the Parker/Fields radio observations [9] should greatly increase our understanding of electron acceleration. 


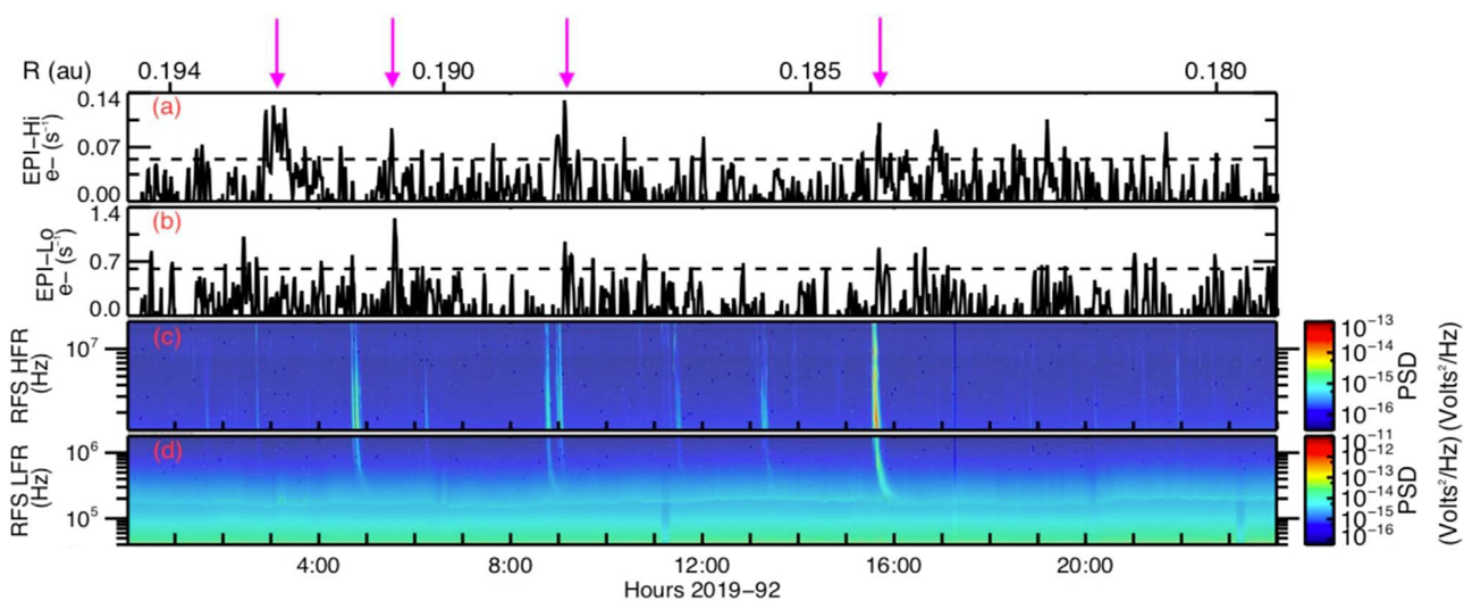

Figure 4. Electron events on 2019 DOY 92. The top two panels are electron fluxes from EPI-Hi $(0.5-6 \mathrm{MeV})$ and EPI-Lo $(50-500 \mathrm{keV})$, and the bottom two panels are radio measurements from Parker/FIELDS.

\section{Data Availability}

The IS@IS data are made public as soon as they are verified, although the processing is still improving as we gain more experience with the instrument and data. Scientists interested in using the data should read the release notes, and it is strongly recommended that those doing detailed analysis consult with the IS@IS team so that they fully understand any systematics that may still be in the data. The data are available from the Princeton University IS@IS webpage (https://spacephysics.princeton.edu/missions-instruments/isois) or the NASA Space Physics Data Facility (https://spdf.gsfc.nasa.gov). In addition, the IS@IS team is developing visualization tools that can be made available to the community. See the Princeton webpages for more information.

\section{Conclusions}

Although the IS@IS team is still waiting for a larger SEP event while Parker is close to the Sun, there has already been a tremendous amount of science coming out of the instrument suite. We are looking forward to a long and exciting mission of discovery and expect many surprises in these unique observations of solar energetic particles. We welcome the community to join us in these studies.

\section{References}

[1] D. J. McComas, et al., Integrated Science Investigation of the Sun (ISIS): Design of the Energetic Particle Investigation. Space Sci Rev 204, 187-256 (2016). Doi: 10.1007/s11214-014-0059-1

[2] I. G. Richardson, et al., Corotating MeV/amu Ion Enhancements at $-<1$ AU From 1978 to 1986. JGR 29, 13 (1993). Doi: 10.1029/92JA01837

[3] D. J. McComas, et al., Probing the energetic particle environment near the Sun. Nature 576, 223 (2019). Doi: 10.1038/s41586-019-1811-1

[4] C. M. S. Cohen, et al., Energetic Particle Increases Associated with Stream Interaction Regions. ApJ 246, 20 (2020). Doi: 10.3847/1538-4365/ab4c38

[5] M. A. I Van Hollebeke, et al., The radial variation of corotating energetic particle streams in the inner and outer solar system. JGR 83, 4723 (1978). Doi: 10.1029/JA083iA10p04723 
[6] C. J. Joyce, et al., Time evolution of stream interaction region energetic particle spectra in the inner heliosphere. A \& A 650, L5 (2021). Doi: 10.1051/0004-6361/202039330

[7] C. M. S. Cohen, et al., Parker Solar Probe observations of He/H abundance variations in SEP events inside 0.5 au. A \& A 650, A23 (2021). Doi: 10.1051/0004-6361/202039299

[8] J. G. Mitchell, et al., Small Electron Events Observed by Parker Solar Probe/ISœIS during Encounter 2. ApJ 902, 20 (2020). Doi: 10.3847/1538-4357/abb2a4

[9] S. D. Bale, et al., The FIELDS Instrument Suite for Solar Probe Plus. Measuring the Coronal Plasma and Magnetic Field, Plasma Waves and Turbulence, and Radio Signatures of Solar Transients. SSR 204, 49 (2016). Doi: 10.1007/s11214-016-0244-5 